\title{
Entangling power and operator entanglement in qudit systems
}

\author{
Xiaoguang Wang, Barry C Sanders, and Dominic W Berry \\ Department of Physics and Centre for Quantum Computer Technology, \\ Macquarie University, Sydney, New South Wales 2109, Australia.
}

(Dated: March 22, 2018)

\begin{abstract}
We establish the entangling power of a unitary operator on a general finite-dimensional bipartite quantum system with and without ancillas, and give relations between the entangling power based on the von Neumann entropy and the entangling power based on the linear entropy. Significantly, we demonstrate that the entangling power of a general controlled unitary operator acting on two equaldimensional qudits is proportional to the corresponding operator entanglement if linear entropy is adopted as the quantity representing the degree of entanglement. We discuss the entangling power and operator entanglement of three representative quantum gates on qudits: the SUM, double SUM, and SWAP gates.
\end{abstract}

PACS numbers: 03.67.Mn, 03.65.Ud

\section{INTRODUCTION}

Entanglement has been established as a crucial resource for quantum information tasks such as quantum communication and quantum computation [1]. Consequently, generating or enhancing entanglement between separate physical systems is of paramount importance in quantum information theory, and two cases are typically studied: (i) ancilla-assisted entanglement generation, and (ii) entanglement generation without assistance from ancillas. Significant effort is currently directed to quantifying entanglement of states; similarly it is important to quantify entanglement capabilities [2, 3, 4, 5, 6, 7, 8, 9, 10] of unitary operations, or more generally, the "strength" 11] of the operator.

Entangling power based on the linear entropy [2] is a valuable, and relatively easy to calculate, measure of the entanglement capability of an operator. We extend this definition to the ancilla-assisted case, and establish an equivalence between entangling power and an alternative quantity, "operator entanglement" 12, 13, for arbitrary controlled unitary operations acting on two equaldimensional qudits.

Any gate that creates entanglement between qudits without ancillas acts as a universal gate for quantum computation when assisted by arbitrary one-qudit gates [14, 15]. Therefore, the SUM gate [16, 17, 18, 19] [a generalization of the controlled-NOT (CNOT) gate for qubits] can be chosen as the basic, or primitive, two-qudit gate for qudit-based quantum computation. We study entangling power of the SUM gate and other two-qudit gates, namely the double-SUM (DSUM) and SWAP gate to illustrate our results on more general gates as well as the general applicability of our approach.

This paper is organized as follows. In Sec. III we introduce the von Neumann entropy and linear entropy as entanglement measures. In Sec. III we review the entangling power based on the linear entropy without ancillas, and extend to entangling power assisted by ancillas. We also give relations between the entangling power based on the von Neumann entropy and the entangling power based on the linear entropy. In Sec. IV we study the entangling power of a general controlled unitary operaror $C_{U}$, and build an equivalence relation between entangling power and operator entanglement. We also provide an example of $C_{U}$ resulting from higher-order spin-spin interactions. In Sec. $\mathbb{\nabla}$ we discuss entangling capabilities of representative two-qudit gates, including SUM [16, 17, 18, 19], DSUM and SWAP gates, and summarize our results in Sec. VI]

\section{ENTANGLEMENT MEASURES}

Various measures of entanglement exist, each with its own advantages and disadvantages $[\underline{6}$. Two commonly used entanglement measures for pure states are the von Neumann entropy $\tilde{E}$ and the linear entropy $E$. For a two-qudit pure state $|\Psi\rangle \in \mathcal{H}_{d} \otimes \mathcal{H}_{d}$ they are defined as

$$
\begin{aligned}
& \tilde{E}(|\Psi\rangle):=-\operatorname{Tr}_{1}\left[\rho_{1} \ln \rho_{1}\right], \\
& E(|\Psi\rangle):=\operatorname{Tr}_{1}\left[\rho_{1}\left(1-\rho_{1}\right)\right]=1-\operatorname{Tr}_{1} \rho_{1}^{2},
\end{aligned}
$$

where $\rho_{1}=\operatorname{Tr}_{2}(|\Psi\rangle\langle\Psi|)$ is the reduced density matrix. For convenience, we use natural logarithms throughout this paper. The von Neumann entropy that we define therefore differs from the usual von Neumann entropy by a factor of $\ln 2$. The von Neumann entropy and the linear entropy satisfy the inequalities

$$
0 \leq \tilde{E}(|\Psi\rangle) \leq \ln d, \quad 0 \leq E(|\Psi\rangle) \leq 1-1 / d
$$

where the lower (upper) bound is reached if and only if $|\Psi\rangle$ is a product state (maximally entangled state).

The entanglement measures discussed above can also be applied to the study of entanglement of operators 12. An operator can increase entanglement of a state, but an operator can also be considered to be entangled because operators themselves inhabit a Hilbert space. The entanglement of quantum operators is introduced 12] by noting that the linear operators over $\mathcal{H}_{d}$ span a $d^{2}$-dimensional Hilbert space with the scalar product 
between two operators $X$ and $Y$ given by the HilbertSchmidt product $\langle X, Y\rangle:=\operatorname{Tr}\left(X^{\dagger} Y\right)$, and $\|X\|_{\mathrm{HS}}:=$ $\sqrt{\operatorname{Tr}\left(X^{\dagger} X\right)}$. We denote this $d^{2}$-dimensional Hilbert space as $\mathcal{H}_{d^{2}}^{\mathrm{HS}}$. Thus, the operator acting on $\mathcal{H}_{d_{1}} \otimes \mathcal{H}_{d_{2}}$ is a state in the composite Hilbert space $\mathcal{H}_{d_{1}^{2}}^{\mathrm{HS}} \otimes \mathcal{H}_{d_{2}^{2}}^{\mathrm{HS}}$, and the entanglement of an operator $X$ is well-defined [12].

Any operator $O$ (not necessarily unitary) acting on $\mathcal{H}_{d_{1}} \otimes \mathcal{H}_{d_{2}}$ may be Schmidt-decomposed as [1] $O=$ $\sum_{n} s_{n} A_{n} \otimes B_{n}$, where $s_{n} \geq 0$ and $\left\{A_{n}\right\}$ and $\left\{B_{n}\right\}$ are orthonormal operator bases for systems 1 and 2. From the Schmidt form, entanglement measures for a unitary operator $U$ can be determined to be

$$
\begin{aligned}
& \tilde{E}(U)=-\sum_{n} \frac{s_{n}^{2}}{d_{1} d_{2}} \ln \left(\frac{s_{n}^{2}}{d_{1} d_{2}}\right), \\
& E(U)=1-\frac{1}{d_{1}^{2} d_{2}^{2}} \sum_{n} s_{n}^{4},
\end{aligned}
$$

where the factor $1 /\left(d_{1} d_{2}\right)$ arises from normalization of the unitary operator.

\section{ASSISTED AND UNASSISTED ENTANGLING POWERS}

The entangling power of a unitary operator $U$ is defined over $\mathcal{H}_{d_{1}} \otimes \mathcal{H}_{d_{2}}$ as the average entanglement of the state $U\left|\psi_{1}\right\rangle \otimes\left|\psi_{2}\right\rangle$ for product states $\left|\psi_{1}\right\rangle \otimes\left|\psi_{2}\right\rangle \in$ $\mathcal{H}_{d_{1}} \otimes \mathcal{H}_{d_{2}}$. The entangling power $\tilde{e}_{\mathrm{p}}(U)$ based on the von Neumann entropy and $e_{\mathrm{p}}(U)$ based on the linear entropy are given by [2, 20]

$$
\begin{aligned}
& \tilde{e}_{\mathrm{p}}(U)=\int d \mu\left(\psi_{1}, \psi_{2}\right) \tilde{E}\left(U\left|\psi_{1}\right\rangle \otimes\left|\psi_{2}\right\rangle\right), \\
& e_{\mathrm{p}}(U)=\int d \mu\left(\psi_{1}, \psi_{2}\right) E\left(U\left|\psi_{1}\right\rangle \otimes\left|\psi_{2}\right\rangle\right),
\end{aligned}
$$

where $d \mu\left(\psi_{1}, \psi_{2}\right)$ denotes an integral measure over product states.

These two entangling powers are related, and relations between linear entropy and von Neumann entropy have been investigated [21, 22]. Let us first rewrite Eqs. (6) and (7) in the form

$$
\begin{aligned}
& \tilde{e}_{\mathrm{p}}(U)=\int d \mu\left(\psi_{1}, \psi_{2}\right) \sum_{i}\left[-\lambda_{i}\left(\psi_{1}, \psi_{2}\right) \ln \lambda_{i}\left(\psi_{1}, \psi_{2}\right)\right] \\
& e_{\mathrm{p}}(U)=1-\int d \mu\left(\psi_{1}, \psi_{2}\right) \sum_{i} \lambda_{i}\left(\psi_{1}, \psi_{2}\right)^{2}
\end{aligned}
$$

where $\lambda_{i}\left(\psi_{1}, \psi_{2}\right)$ are the squares of the coefficients in the Schmidt decomposition of $U\left|\psi_{1}\right\rangle \otimes\left|\psi_{2}\right\rangle$. In addition, let us define the entangling power

$$
\bar{e}_{\mathrm{p}}(U)=-\ln \left(1-e_{\mathrm{p}}(U)\right),
$$

which is a monotonic function of the entangling power $e_{\mathrm{p}}(U)$, and satisfies $\bar{e}_{\mathrm{p}}(U) \geq e_{\mathrm{p}}(U)$.
Evaluating $\tilde{e}_{\mathrm{p}}(U)-\bar{e}_{\mathrm{p}}(U)$ gives

$$
\begin{aligned}
& \tilde{e}_{\mathrm{p}}(U)-\bar{e}_{\mathrm{p}}(U) \\
& =\int d \mu\left(\psi_{1}, \psi_{2}\right) \sum_{i}\left[-\lambda_{i}\left(\psi_{1}, \psi_{2}\right) \ln \frac{\lambda_{i}\left(\psi_{1}, \psi_{2}\right)}{1-e_{\mathrm{p}}(U)}\right] \\
& \geq \int d \mu\left(\psi_{1}, \psi_{2}\right) \sum_{i} \lambda_{i}\left(\psi_{1}, \psi_{2}\right)\left[1-\frac{\lambda_{i}\left(\psi_{1}, \psi_{2}\right)}{1-e_{\mathrm{p}}(U)}\right] \\
& =1-\frac{1}{1-e_{\mathrm{p}}(U)} \int d \mu\left(\psi_{1}, \psi_{2}\right) \sum_{i} \lambda_{i}\left(\psi_{1}, \psi_{2}\right)^{2} \\
& =0 .
\end{aligned}
$$

This result implies that $\tilde{e}_{\mathrm{p}}(U) \geq \bar{e}_{\mathrm{p}}(U)$. Another useful bound on $\tilde{e}_{\mathrm{p}}(U)$ can be obtained by noting that the average entanglement generation cannot be larger than the maximum entanglement generation: $\tilde{e}_{\mathrm{p}}(U) \leq \tilde{E}_{\max }(U)$, where

$$
\tilde{E}_{\max }(U)=\max _{\left|\psi_{1}\right\rangle,\left|\psi_{2}\right\rangle} \tilde{E}\left(U\left|\psi_{1}\right\rangle \otimes\left|\psi_{2}\right\rangle\right)
$$

These relations are useful because $\bar{e}_{\mathrm{p}}(U)$ and $\tilde{E}_{\max }(U)$ may be determined analytically, and used to draw conclusions about $\tilde{e}_{\mathrm{p}}(U)$.

Now we investigate the entangling power based on the linear entropy. The calculation of linear entropy $E$ can be simplified by doubling the Hilbert space from $\mathcal{H}_{d_{1}} \otimes$ $\mathcal{H}_{d_{2}}$ to $\mathcal{H}_{d_{1}} \otimes \mathcal{H}_{d_{2}} \otimes \mathcal{H}_{d_{1}} \otimes \mathcal{H}_{d_{2}}$ and using the identity $\operatorname{Tr}_{12}\left[(\hat{A} \otimes \hat{B}) S_{12}\right]=\operatorname{Tr}_{1}(\hat{A} \hat{B})[2]$. Here $S_{i j}$ denotes the swap operation between equal-dimensional systems $i$ and $j$. It is clear from Eq. (7) that different integral measures give different entangling powers. For the Haar measure, group theory techniques yield [2]

$$
\begin{aligned}
e_{\mathrm{p}}(U)= & 1-\frac{1}{d_{1}\left(d_{1}+1\right) d_{2}\left(d_{2}+1\right)}\left[d_{1} d_{2}^{2}+d_{2} d_{1}^{2}\right. \\
& +\operatorname{Tr}_{1234}\left(U^{\otimes 2} S_{13} U^{\dagger \otimes 2} S_{13}\right) \\
& \left.+\operatorname{Tr}_{1234}\left(U^{\otimes 2} S_{24} U^{\dagger \otimes 2} S_{13}\right)\right] .
\end{aligned}
$$

This definition of entangling power presents an anomaly that the entangling power of a SWAP gate over a $d \times d$ space is zero [2]. The entangling power defined by Eq. (7) does not include the advantage of incorporating ancilla assistance. With assistance from ancillas the SWAP gate can generate entanglement. The dimension of each ancilla can be chosen as the dimension of the original system because the Schmidt number of a state in the composite system of the original system plus ancilla is at most the dimension of the original system 11.

Let the SWAP gate for systems $A$ and $B$ act on the state $|\Psi\rangle_{A^{\prime} A} \otimes|\Phi\rangle_{B B^{\prime}}$, where

$$
\begin{aligned}
|\Psi\rangle_{A^{\prime} A} & =\sum_{n=0}^{d-1}|n\rangle_{A^{\prime}} \otimes|n\rangle_{A} \in \mathcal{H}_{d} \otimes \mathcal{H}_{d}, \\
|\Phi\rangle_{B B^{\prime}} & =\sum_{n=0}^{d-1}|n\rangle_{B} \otimes|n\rangle_{B^{\prime}} \in \mathcal{H}_{d} \otimes \mathcal{H}_{d},
\end{aligned}
$$


and $A^{\prime}$ and $B^{\prime}$ denote ancillas for $A$ and $B$, respectively. The final state after applying $U$ will have entanglement increased by $E=1-1 / d^{2}$. The entanglement increase draws on the ancillary resources. Without these ancillas, the SWAP gate cannot increase entanglement, which is the case considered in Ref. [2].

We consider a $d_{1} \times d_{2}$ system, and introduce two ancillas $A^{\prime}$ and $B^{\prime}$ with dimension $d_{1}$ and $d_{2}$, respectively. Then, the whole state space expands to $\mathcal{H}_{d_{1}}^{\otimes 2} \otimes \mathcal{H}_{d_{2}}^{\otimes 2}$ in which the first and fourth systems are ancillas. Let the unitary operator $U$ act on the whole state space $\mathcal{H}_{d_{1}}^{\otimes 2} \otimes \mathcal{H}_{d_{2}}^{\otimes 2}$. Analogous to Eq. (7), we define the ancillaassisted entangling power as

$$
e_{\mathrm{p}}^{\mathrm{anc}}(U)=\int d \mu(\alpha, \beta) E\left(U|\alpha\rangle_{12} \otimes|\beta\rangle_{34}\right),
$$

where $|\alpha\rangle_{12} \in \mathcal{H}_{d_{1}}^{\otimes 2}$ and $|\beta\rangle_{34} \in \mathcal{H}_{d_{2}}^{\otimes 2}$. By splitting the whole system as subsystems 12 and 34, extending Eq. (13), and using the Haar measure, we obtain the assisted entangling power as

$$
\begin{aligned}
e_{\mathrm{p}}^{\mathrm{anc}}(U)= & 1-\frac{1}{d_{1}^{2}\left(d_{1}^{2}+1\right) d_{2}^{2}\left(d_{2}^{2}+1\right)}\left\{d_{1}^{2} d_{2}^{4}+d_{2}^{2} d_{1}^{4}\right. \\
& +\operatorname{Tr}_{12 \ldots 8}\left[U^{\otimes 2}\left(S_{15} S_{26}\right) U^{\dagger \otimes 2}\left(S_{15} S_{26}\right)\right] \\
& \left.+\operatorname{Tr}_{12 \ldots 8}\left[U^{\otimes 2}\left(S_{37} S_{48}\right) U^{\dagger \otimes 2}\left(S_{37} S_{48}\right)\right]\right\}
\end{aligned}
$$

where the state space now involved has doubled to $\mathcal{H}_{d_{1}}^{\otimes 2} \otimes$ $\mathcal{H}_{d_{2}}^{\otimes 2} \otimes \mathcal{H}_{d_{1}}^{\otimes 2} \otimes \mathcal{H}_{d_{2}}^{\otimes 2}$. Operator $S_{i j} S_{k l}$ is the swap between systems $i$ and $k$ and systems $j$ and $l$. We are interested only in the case that the unitary operator acts on the system and not the ancillas, i.e., $U \equiv I_{14} \otimes U_{23}$. Equation (17) enables the calculation of the assisted entangling power of $U$.

Qudit quantum computation is normally considered for many qudits with equal dimension [14]. We will mainly examine the entangling powers of two-qudit quantum gates as building blocks of the quantum computer, and therefore we restrict to the case of equal dimension [5] $\left(d_{1}=d_{2}\right)$. In this case it is found that the entangling power of a unitary operator $U$ is related to the entanglement of quantum unitary operators [12, 13]. The operator entanglement of unitary operator $U$ is given by 12

$$
E(U)=1-\frac{1}{d^{4}} \operatorname{Tr}\left(U^{\otimes 2} S_{13} U^{\dagger \otimes 2} S_{13}\right),
$$

where $1 / d^{4}$ is just the normalization factor for $U^{\otimes 2}$. From Eqs. (13) and (18), it is straightforward to verify [2]

$$
e_{\mathrm{p}}(U)=\left(\frac{d}{d+1}\right)^{2}\left[E(U)+E\left(U S_{12}\right)-E\left(S_{12}\right)\right] .
$$

Thus, the unassisted entangling power defined on $d \times d$ systems can be expressed in terms of the entanglement of three operators, $U, U S_{12}$, and $S_{12}$. Therefore, by studying the entanglement of these three operators we can determine the entangling power of $U$.
From Eqs. (17) and (18), a similar result can be obtained for the assisted entangling power as follows

$$
\begin{aligned}
e_{\mathrm{p}}^{\mathrm{anc}}(U)= & \left(\frac{d^{2}}{d^{2}+1}\right)^{2} \\
& \times\left[E(U)+E\left(U S_{13} S_{24}\right)-E\left(S_{13} S_{24}\right)\right] .
\end{aligned}
$$

Note that relations (19) and (20) hold only when we quantify the entanglement by the linear entropy, and from these relations we know that unassisted and assisted entangling powers are completely determined by the operator entanglement of $U, U S_{12}$, and $U S_{13} S_{24}$ (the entangling powers of $S_{12}$ and $S_{13} S_{24}$ are given below). Based on these results for the entangling powers, we next investigate a general controlled- $U$ quantum operation on qudits.

\section{A GENERAL TWO-QUDIT CONTROLLED- $U$ GATE}

A general controlled- $U$ quantum operation on two qudits is given by

$$
C_{U}:=\sum_{n=0}^{d-1} P_{n, n} \otimes U_{n}
$$

with $P_{n, n}:=|n\rangle\langle n|$. We also define $P_{n, m}:=|n\rangle\langle m|$, which satisfies $P_{n, m} P_{k, l}=\delta_{m k} P_{n, l}$. The controlled- $U$ gate implements the unitary operator $U_{n}$ on the second system if and only if the first system is in the state $|n\rangle$. The unassisted entangling power and operator entanglement have been computed for the $C_{U}$ with $d$ orthogonal $U_{n}$ [2, 12]. Here, $U_{n}$ can be arbitrary unitary operators. For the controlled- $U$ operation we have the following proposition.

Proposition 1: For the general controlled- $U$ gate acting on $\mathcal{H}_{d} \otimes \mathcal{H}_{d}$

$$
\begin{aligned}
e_{\mathrm{p}}\left(C_{U}\right) & =\left(\frac{d}{d+1}\right)^{2} E\left(C_{U}\right), \\
e_{\mathrm{p}}^{\mathrm{anc}}\left(C_{U}\right) & =\left(\frac{d^{2}}{d^{2}+1}\right)^{2} E\left(C_{U}\right) .
\end{aligned}
$$

Proof: From Eqs. (19) and (20), we only need to prove that

$$
E\left(C_{U} S_{12}\right)=E\left(S_{12}\right), E\left(C_{U} S_{13} S_{24}\right)=E\left(S_{13} S_{24}\right) .
$$

Let us first prove $E\left(C_{U} S_{12}\right)=E\left(S_{12}\right)$. The swap operator $S_{12}$ can be written as

$$
S_{12}=\sum_{i=j=0}^{d-1} P_{i, j} \otimes P_{j, i}
$$

It is easy to check that $\left\langle P_{i, j}, P_{k, l}\right\rangle=\operatorname{Tr}\left(P_{j, i} P_{k, l}\right)=\delta_{i k} \delta_{j l}$. Therefore, $S_{12}$ is in the Schmidt form with Schmidt number $d^{2}$, and the operator entanglement is given by

$$
E\left(S_{12}\right)=1-1 / d^{2} \text {. }
$$


From Eqs. (21) and (25), we write the product of the operators $C_{U}$ and $S_{12}$ as

$$
C_{U} S_{12}=\sum_{i, j} P_{i, j} \otimes U_{i} P_{j, i}
$$

The operator product $C_{U} S_{12}$ is also in the Schmidt form with Schmidt number $d^{2}$ since

$$
\left\langle U_{i} P_{j, i}, U_{k} P_{l, k}\right\rangle=\operatorname{Tr}\left(P_{i, j} U_{i}^{\dagger} U_{k} P_{l, k}\right)=\delta_{i k} \delta_{j l} .
$$

Thus, the operator entanglement is

$$
E\left(C_{U} S_{12}\right)=E\left(S_{12}\right)=1-1 / d^{2},
$$

which complete the proof of Eq. (22).

To prove Eq. (23) we write

$$
S_{13} S_{24}=\sum_{i j k l}\left(P_{i, j} \otimes P_{k, l}\right) \otimes\left(P_{j, i} \otimes P_{l, k}\right) .
$$

Then the operator product $C_{U} S_{13} S_{24}$ is given by

$$
C_{U} S_{13} S_{24}=\sum_{i j k l}\left(P_{i, j} \otimes P_{k, l}\right) \otimes\left(U_{k} P_{j, i} \otimes P_{l, k}\right) .
$$

It is straightforward to check that operators $S_{13} S_{24}$ and $C_{U} S_{13} S_{24}$ are in equivalent Schmidt forms; hence their entanglements are equal, i.e.,

$$
E\left(C_{U} S_{13} S_{24}\right)=E\left(S_{13} S_{24}\right)=1-1 / d^{4} .
$$

This completes the proof of Eq. (23).

Proposition 1 builds an equivalence relation between entangling powers and operator entanglement. The higher the operator entanglement, the higher the entangling powers are for the general $C_{U}$ gate. From Eqs. (22) and (23), we immediately find

$$
\frac{e_{\mathrm{p}}^{\mathrm{anc}}\left(C_{U}\right)}{e_{\mathrm{p}}\left(C_{U}\right)}=\left(\frac{d^{2}+d}{d^{2}+1}\right)^{2}>1,
$$

which means that the entangling power of $C_{U}$ is enhanced by introducing ancillas.

We now apply Proposition 1 to study an example of a $C_{U}$ gate, and we will see that the controlled-PHASE (CPHASE) gate 16] is a special case of this $C_{U}$ gate. We consider the interaction between two spin- $j$ systems via the Hamiltonian 23.

$$
H=g J_{1 z} \otimes J_{2 z},
$$

with $g$ the coupling strength and $J_{i z}$ the $z$-component of the angular momentum operator $\vec{J}_{i}$. Up to local unitary operations, the evolution operator $\exp \left(-i g t J_{1 z} \otimes J_{2 z}\right)$ is equivalent to $U(\theta)=e^{i \theta N_{1} \otimes N_{2}}$, where $N_{i}=J_{i z}+j$ and $\theta=-g t$. Note that the above unitary operator can be written as $U(\theta)=\sum_{n=0}^{d-1} P_{n, n} \otimes e^{i n \theta N_{2}}$, where $d=2 j+1$. Then the unitary operator $U(\theta)$ is a special case of the controlled- $U$ gate. The application of Proposition 1 to $U(\theta)$ tells us that the entangling power $e_{\mathrm{p}}$

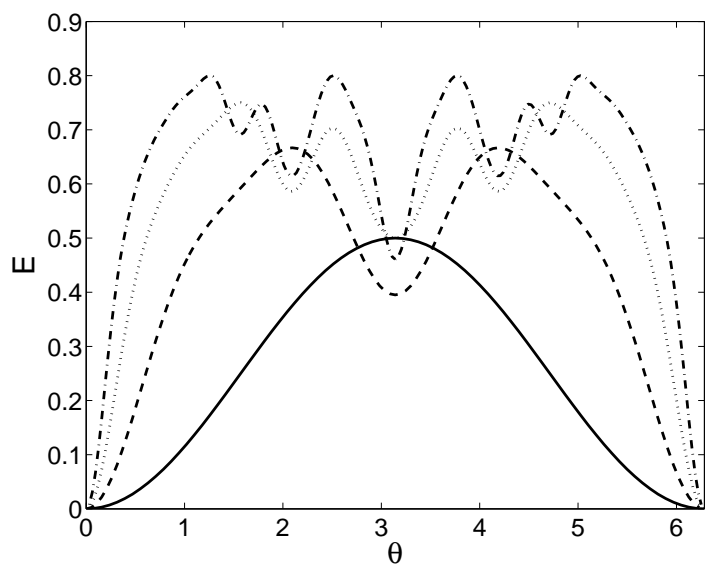

FIG. 1: The entanglement of the operator $U(\theta)$ vs $\theta$ for four different spins: spin-1/2 (solid line), spin-1 (dashed line), spin-3/2 (dotted line), and spin-2 (dash-dot line).

and $e_{\mathrm{p}}^{\text {anc }}$ are proportional to the operator entanglement of $U(\theta)$. Thus, we only need to calculate the operator entanglement.

The unitary operator $U(\theta)$ can be rewritten as

$$
U(\theta)=\sum_{m, n=0}^{d-1} \frac{1}{d} e^{i \theta m n} P_{n, n} \otimes P_{m, m},
$$

where $1 / d$ is just the normalization factor. We consider the operators $U$ and $P_{n, n}$ as states $|U\rangle$ and $\left|P_{n, n}\right\rangle$, where the bra-ket formalism is used. After tracing out the second system we obtain the "mixed operator" for the first system,

$$
\operatorname{Tr}_{2}(|U\rangle\langle U|)=\sum_{m n} A_{m n}(\theta)\left|P_{m, m}\right\rangle\left\langle P_{n, n}\right|,
$$

with

$$
\begin{aligned}
A_{m n}(\theta) & =\frac{1}{d^{2}} \sum_{k=0}^{d-1} e^{i \theta k(m-n)} \\
& =\frac{1}{d^{2}} \frac{\sin [d \theta(m-n) / 2]}{\sin [\theta(m-n) / 2]} e^{i(d-1) \theta(m-n) / 2} .
\end{aligned}
$$

For the case of two spin- $1 / 2$ systems it is straightforward to check that $E(U)=1 / 2 \sin ^{2}(\theta / 2)[12$. For higher spins we need to find the eigenvalues of the $d \times d$ matrix $A$, from which the linear entropy can be obtained. We numerically diagonalize the matrix, and the results for the linear entropy are shown in Fig. 1]

From Fig. [1 we see that the entanglement is a periodic function of $\theta$ with period $2 \pi$, which can also be seen from Eq. (37). The entanglement attains its maximum value of $1 / 2$ at $\theta=\pi$ for spin- $1 / 2$, but does not reach its maximum value $1-1 / d$ at $\theta=\pi$ for spins greater than $1 / 2$. The first maximum value occurs at $\theta=2 \pi / d$. We also observe that there are two maximum values in one period for spin 1 and spin $3 / 2$ and four for spin 2 . 
When $\theta=2 \pi / d$ the unitary operator $U(2 \pi / d)$ becomes the CPHASE gate on qudits [16]. Detailed analysis of the operator entanglement for the CPHASE gate and other representative quantum gates is provided in Sec. $\mathbf{\nabla}$

\section{ENTANGLEMENT CAPABILITY OF QUDIT GATES}

A qudit quantum computer is comprised of a network of one-qudit, two-qudit, and multi-qudit gates. Twoqudit or multi-qudit gates usually have entanglement capability. In this section, we calculate and compare entanglement capabilities of different two-qudit gates. Before going to entangling gates let us first review several useful one-qudit gates.

\section{A. One-Qudit gates}

Two essential one-qudit gates, denoted by $X$ and $Z$, are defined by their action on the computational basis $|n\rangle(n=0, \ldots, d-1)$

$$
X|n\rangle=|n+1(\bmod d)\rangle, \quad Z|n\rangle=\exp (i 2 \pi n / d)|n\rangle .
$$

Another useful quantum operation on qudits is the Fourier transformation $F$, which is defined as

$$
F|n\rangle=\sum_{k=0}^{d-1} \exp (i 2 n k \pi / d)|k\rangle .
$$

The Fourier transformation reduces to the Hadamard gate for the case of $d=2$.

\section{B. The CPHASE and SUM gate}

Henceforth we use $U_{\text {GATE }}$ to denote a two-qudit gate which includes the CPHASE, SUM, DSUM, and SWAP gates. Now we examine the unitary operator $U(\theta)=$ $e^{i \theta \mathcal{N}_{1} \otimes \mathcal{N}_{2}}$ again. Note that the number operator $\mathcal{N}$ is now simply defined as $\mathcal{N}|n\rangle=n|n\rangle$. For $\theta=2 \pi / d, U(\theta)$ can be written as

$$
U_{\mathrm{CPHASE}}=U(2 \pi / d)=\sum_{n=0}^{d-1} P_{n, n} \otimes Z^{n},
$$

which is exactly the CPHASE gate [16]. We will see that the CPHASE gate differs from the SUM gate (defined below) only by local operations.

Let us consider one representative two-qudit gate, namely the SUM gate, which is defined as 16, 17, 18, 19]

$$
U_{\mathrm{SUM}}=U_{\mathrm{SUM}}(1 \rightarrow 2):=\sum_{n=0}^{d-1} P_{n, n} \otimes X^{n}
$$

The notation $(1 \rightarrow 2)$ indicates that the first qudit is the control and the second qudit is the target. By using the Fourier transform we have $F^{-1} Z F=X$. Then, acting on the CPHASE gate $U_{\mathrm{CPHASE}}$ by $I \otimes F^{-1}$ from the left and $I \otimes F$ from the right leads to the relation between the CPHASE gate and the SUM gate,

$$
U_{\mathrm{SUM}}=\left(I \otimes F^{-1}\right) U_{\mathrm{CPHASE}}(I \otimes F) .
$$

Relation (42) shows that the SUM and CPHASE gates differ only by local unitary operations. Therefore, they have same operator entanglement and entangling powers. The SUM gate is an example of a general controlled- $U$ gate, and it has Schmidt form

$$
U_{\mathrm{SUM}}=\sum_{n=0}^{d-1} \sqrt{d} P_{n, n} \otimes\left(X^{n} / \sqrt{d}\right) .
$$

Thus, the entanglement of the SUM gate is given by

$$
E\left(U_{\mathrm{SUM}}\right)=1-1 / d
$$

According to Proposition 1 the unassisted and assisted entangling powers are immediately evident.

\section{The SWAP gate}

Another representative quantum gate is the SWAP gate $U_{\mathrm{SWAP}}$, which we have denoted by SWAP and $S_{i j}$ in the preceding sections. We know that $E\left(U_{\mathrm{SWAP}}\right)=$ $1-1 / d^{2}$; now, from Eq. (19), it is easy to ascertain that $e_{\mathrm{p}}\left(U_{\mathrm{SWAP}}\right)=0$.

Now we calculate the assisted entangling power of the SWAP gate. As we already know the entanglement of operators $S_{23}$ and $S_{13} S_{24}$, only the entanglement of the operator $S_{23} S_{13} S_{24}$ needs to be calculated. The operator $S_{23} S_{13} S_{24}$ can be expressed as

$$
\begin{aligned}
S_{23} S_{13} S_{24} & =\sum_{m n i j k l}\left(P_{i, j} \otimes P_{m, n} P_{k, l}\right) \otimes\left(P_{n, m} P_{j, i} \otimes P_{l, k}\right) \\
& =\sum_{i l} \mathcal{P}_{i, l} \otimes \mathcal{P}_{i, l}^{\dagger},
\end{aligned}
$$

with $\mathcal{P}_{i, l}=\sum_{j} P_{i, j} \otimes P_{j, l}$ satisfying the relations

$$
\left\langle\mathcal{P}_{i, l}, \mathcal{P}_{i^{\prime}, l^{\prime}}\right\rangle=d \delta_{i i^{\prime}} \delta_{l l^{\prime}}
$$

Therefore, the Schmidt form of $S_{23} S_{13} S_{24}$ is given by

$$
S_{23} S_{13} S_{24}=\sum_{i l} d\left(\mathcal{P}_{i, l} / \sqrt{d}\right) \otimes\left(\mathcal{P}_{i, l}^{\dagger} / \sqrt{d}\right)
$$

from which the operator entanglement of $S_{23} S_{13} S_{24}$ is obtained as

$$
E\left(S_{23} S_{13} S_{24}\right)=E\left(S_{23}\right)=1-1 / d^{2} .
$$


Then, substituting the above equation and Eq. (32) into Eq. (20), we obtain

$$
e_{\mathrm{p}}^{\mathrm{anc}}\left(S_{23}\right)=\left(\frac{d^{2}-1}{d^{2}+1}\right)^{2} .
$$

After introducing ancillas the entangling power of the SWAP gate is no longer zero.

\section{Double SUM gate}

In this subsection we introduce and define a double SUM gate as

$$
U_{\mathrm{DSUM}}=U_{\mathrm{SUM}}^{-1}(2 \rightarrow 1) U_{\mathrm{SUM}}(1 \rightarrow 2)
$$

which can be considered as a generalization of the double CNOT gate for qubits in the sense that the DSUM gate reduces to the double controlled-NOT gate [24, 25] for the case of dimension $d=2$.

Using the relation between SWAP and SUM gates given by [26, 27, 28]

$$
\begin{aligned}
S_{12} & =\left(F^{2} \otimes I\right) U_{\mathrm{SUM}}(1 \rightarrow 2) U_{\mathrm{SUM}}^{-1}(2 \rightarrow 1) U_{\mathrm{SUM}}(1 \rightarrow 2) \\
& =\left(F^{2} \otimes I\right) U_{\mathrm{SUM}} U_{\mathrm{DSUM}},
\end{aligned}
$$

we observe that the SWAP gate can be constructed from three SUM gates and the square of the Fourier transformation. This relation is useful for the following analysis.

By using Eq. (51) we can express DSUM as

$$
U_{\mathrm{DSUM}}=U_{\mathrm{SUM}}^{-1}(1 \rightarrow 2) S_{12}\left(I \otimes F^{2}\right),
$$

where the identity $(\hat{A} \otimes \hat{B}) S_{12}=S_{12}(\hat{B} \otimes \hat{A})$ is used. From Proposition 1, we know that for any controlled- $U$ we have $E\left(C_{U} S_{12}\right)=E\left(S_{12}\right)$. As $U_{\mathrm{SUM}}^{-1}(1 \rightarrow 2)$ is a special $C_{U}$ with $U=X^{-1}$, we have

$$
\begin{aligned}
E\left(U_{\mathrm{DSUM}}\right) & =E\left[U_{\mathrm{SUM}}^{-1}(1 \rightarrow 2) S_{12}\left(I \otimes F^{2}\right)\right] \\
& =E\left[U_{\mathrm{SUM}}^{-1}(1 \rightarrow 2) S_{12}\right]=E\left(S_{12}\right),
\end{aligned}
$$

where the second equality is obtained by noticing that the local unitary operators do not modify operator entanglement. Thus, we find that the entanglement of the DSUM gate is equal to that of the SWAP gate. Using this fact the unassisted entangling power is simplified to

$$
\begin{aligned}
e_{\mathrm{p}}\left(U_{\mathrm{DSUM}}\right) & =\frac{d^{2}}{(d+1)^{2}} E\left[U_{\mathrm{SUM}}^{-1}(1 \rightarrow 2) S_{12}\left(I \otimes F^{2}\right) S_{12}\right] \\
& =\frac{d^{2}}{(d+1)^{2}} E\left[U_{\mathrm{SUM}}^{-1}(1 \rightarrow 2)\right] \\
& =e_{\mathrm{p}}\left[U_{\mathrm{SUM}}(1 \rightarrow 2)\right] .
\end{aligned}
$$

The last equality in the above equation results from the fact $E(U)=E\left(U^{\dagger}\right)$ 12. Therefore, the unassisted entangling power of the DSUM gate is equal to that of the SUM gate.
TABLE I: Entangling powers $e_{\mathrm{p}}, e_{\mathrm{p}}^{\text {anc }}$, and operator entanglement $E$ for the three representative two-qudit gates.

\begin{tabular}{cccc} 
Gates & $e_{\mathrm{p}}$ & $e_{\mathrm{p}}^{\text {anc }}$ & $E$ \\
\hline$U_{\text {SUM }}$ & $\frac{d(d-1)}{(d+1)^{2}}$ & $\frac{d^{3}(d-1)}{\left(d^{2}+1\right)^{2}}$ & $1-1 / d$ \\
\hline$U_{\text {DSUM }}$ & $\frac{d(d-1)}{(d+1)^{2}}$ & $\frac{d^{4}-d^{2}-d+1}{\left(d^{2}+1\right)^{2}}$ & $1-1 / d^{2}$ \\
\hline$U_{\text {SWAP }}$ & 0 & $\frac{\left(d^{2}-1\right)^{2}}{\left(d^{2}+1\right)^{2}}$ & $1-1 / d^{2}$ \\
\hline
\end{tabular}

To obtain the assisted entangling power of the DSUM gate we need to calculate the entanglement of the operator $U_{\mathrm{DSUM}} S_{13} S_{24}$. Up to local unitary operations the operator is equivalent to $U_{\mathrm{SUM}}^{-1}(1 \rightarrow 2) S_{23} S_{13} S_{24}$, which can be expressed as

$$
\begin{aligned}
& U_{\mathrm{SUM}}^{-1}(1 \rightarrow 2) S_{23} S_{13} S_{24} \\
& =\sum_{i m l}\left(P_{m, i} \otimes P_{i, l}\right) \otimes\left(\sum_{j} P_{j-i, m} \otimes P_{l, j}\right) .
\end{aligned}
$$

It is straightforward to show the relations

$$
\begin{aligned}
\left\langle P_{m, i} \otimes P_{i, l}, P_{m^{\prime}, i^{\prime}} \otimes P_{i^{\prime}, l^{\prime}}\right\rangle & =\delta_{m m^{\prime}} \delta_{l l^{\prime}} \delta_{i i^{\prime}}, \\
\left\langle\sum_{j} P_{j-i, m} \otimes P_{l, j}, \sum_{j^{\prime}} P_{j^{\prime}-i^{\prime}, m^{\prime}} \otimes P_{l^{\prime}, j^{\prime}}\right\rangle & =d \delta_{m m^{\prime}} \delta_{l l^{\prime}} \delta_{i i^{\prime}} .
\end{aligned}
$$

Thus, $U_{\text {SUM }}^{-1}(1 \rightarrow 2) S_{23} S_{13} S_{24}$ can be written in the Schmidt form and the entanglement

$$
E\left(U_{\mathrm{DSUM}} S_{13} S_{24}\right)=1-1 / d^{3}
$$

follows. Using the above equation, $E\left(U_{\mathrm{DSUM}}\right)=1-$ $1 / d^{2}$, and $E\left(S_{13} S_{24}\right)=1-1 / d^{4}$, we obtain the assisted entangling power of the double SUM gate as

$$
e_{\mathrm{p}}^{\mathrm{anc}}\left(U_{\mathrm{DSUM}}\right)=\frac{d^{4}-d^{2}-d+1}{\left(d^{2}+1\right)^{2}} .
$$

We summarize the results of the three representative quantum gates by Table \In particular, the two equalities

$$
E\left(U_{\mathrm{SWAP}}\right)=E\left(U_{\mathrm{DSUM}}\right), e_{\mathrm{p}}\left(U_{\mathrm{SUM}}\right)=e_{\mathrm{p}}\left(U_{\mathrm{DSUM}}\right),
$$

hold. As the entangling power $e_{\mathrm{p}}$ of the DSUM gate is not zero, we can use it as a universal gate in a qudit quantum computer. Although the operator entanglement of the SWAP gate is equal to that of the DSUM gate, we cannot use the SWAP gate as a universal gate since the corresponding entangling power $e_{\mathrm{p}}$ is zero.

\section{E. Large dimension limit}

Now we consider the large $d$ limit. In this limit, we find that in every case (except for the SWAP without ancillas) the values of $e_{\mathrm{p}}$ and $e_{\mathrm{p}}^{\text {anc }}$ approach 1 . It is therefore better 
TABLE II: Asymptotic expressions for the entangling powers $\bar{e}_{\mathrm{p}}$ and $\bar{e}_{\mathrm{p}}^{\mathrm{anc}}$, for the three representative two-qudit gates.

\begin{tabular}{ccc} 
Gates & $\bar{e}_{\mathrm{p}}$ & $\bar{e}_{\mathrm{p}}^{\text {anc }}$ \\
\hline$U_{\mathrm{SUM}} \ln d-\ln 3+O\left(d^{-1}\right)$ & $\ln d+O\left(d^{-1}\right)$ \\
\hline$U_{\mathrm{DSUM}} \ln d-\ln 3+O\left(d^{-1}\right)$ & $2 \ln d-\ln 3+O\left(d^{-1}\right)$ \\
\hline$U_{\text {SWAP }}$ & 0 & $2 \ln d-\ln 4+O\left(d^{-2}\right)$ \\
\hline
\end{tabular}

TABLE III: Asymptotic expressions for the entangling powers based on the von Neumann entropy, $\tilde{e}_{\mathrm{p}}$ and $\tilde{e}_{\mathrm{p}}^{\text {anc }}$, for the three representative two-qudit gates.

\begin{tabular}{ccc} 
Gates & $\tilde{e}_{\mathrm{p}}$ & $\tilde{e}_{\mathrm{p}}^{\text {anc }}$ \\
\hline$U_{\mathrm{SUM}}$ & $\ln d+O(1)$ & $\ln d+O\left(d^{-1}\right)$ \\
\hline$U_{\text {DSUM }}$ & $\ln d+O(1)$ & $2 \ln d+O(1)$ \\
\hline$U_{\mathrm{SWAP}}$ & 0 & $2 \ln d+O(1)$ \\
\hline
\end{tabular}

to consider the measure $\bar{e}_{\mathrm{p}}$ (10), for the case without ancillas, and $\bar{e}_{\mathrm{p}}^{\mathrm{anc}}=-\ln \left(1-e_{\mathrm{p}}^{\mathrm{anc}}\right)$, for the case with ancillas. The asymptotic expressions for these quantities for the three different gates are given in Table II.

These asymptotic results may be used to gain information about the entangling powers based on the von Neumann entropy, $\tilde{e}_{\mathrm{p}}$ and $\tilde{e}_{\mathrm{p}}^{\text {anc }}$. As was shown above, $\tilde{e}_{\mathrm{p}} \geq \bar{e}_{\mathrm{p}}$, and it is also easily seen that $\tilde{e}_{\mathrm{p}}^{\mathrm{anc}} \geq \bar{e}_{\mathrm{p}}^{\mathrm{anc}}$. In addition, the maximum von Neumann entropy generation for each of the operations [30] is equal to the leading terms in the asymptotic expressions in Table 1 .

These results allow us to accurately estimate the asymptotic values of $\tilde{e}_{\mathrm{p}}$ and $\tilde{e}_{\mathrm{p}}^{\mathrm{anc}}$. For example, for the case of the SUM gate without ancillas,

$$
\ln d-\ln 3+O\left(d^{-1}\right) \leq \tilde{e}_{\mathrm{p}}\left(U_{\mathrm{SUM}}\right) \leq \ln d .
$$

This result means that $\tilde{e}_{\mathrm{p}}\left(U_{\mathrm{SUM}}\right)=\ln d+O(1)$. The corresponding results for the other cases are given in Table IIII In every case, to leading order $\tilde{e}_{\mathrm{p}}^{\mathrm{anc}}, \bar{e}_{\mathrm{p}}^{\mathrm{anc}}$, and the maximal entanglement are the same.

We therefore find that, in each of these cases (except the case of the SWAP without ancillas)

$$
\lim _{d \rightarrow \infty} \frac{\tilde{e}_{\mathrm{p}}}{\bar{e}_{\mathrm{p}}}=\lim _{d \rightarrow \infty} \frac{\tilde{e}_{\mathrm{p}}}{\tilde{E}_{\max }}=1 .
$$

In the case of the SUM gate with ancillas the agreement is particularly close. Because the second term is of order $d^{-1}$, rather than order 1 ,

$$
\lim _{d \rightarrow \infty} \tilde{e}_{\mathrm{p}}^{\mathrm{anc}}\left(U_{\mathrm{SUM}}\right)=\lim _{d \rightarrow \infty} \bar{e}_{\mathrm{p}}^{\mathrm{anc}}\left(U_{\mathrm{SUM}}\right)=\tilde{E}_{\mathrm{max}}\left(U_{\mathrm{SUM}}\right) .
$$

That is, the average entanglement created approaches the maximum possible, rather than just the ratio approaching 1. In addition, the results obtained for the SUM gate is applicable to any controlled- $U$ gate $C_{U}$ (21) with $d$ orthogonal $U_{n}$.

\section{CONCLUSIONS}

In conclusion, we have extended the entangling power based on the linear entropy from the ancilla-unassisted case to the ancilla-assisted case. The assisted and unassisted entangling powers, quantifying the average amount of entanglement created by a unitary operator, turn out to be easy-to-use entanglement capability measures which are complementary to the entanglement capability measures based on the maximal entanglement $6,7,11]$ that an operator can generate.

We have studied the general controlled- $U$ operator and found that both the unassisted and assisted entangling powers are proportional to its operator entanglement, which builds equivalence relations between the entangling power and operator entanglement. This is important because the set of controlled- $U$ gates contains some very useful quantum gates such as the CPHASE and SUM gates, and our result shows that it is sufficient to study the entanglement capability by examining the operator entanglement. From the SUM gate, we have derived a new quantum gate, the DSUM gate, which for qubits reduces to the double CNOT gate. The entangling powers and operator entanglement of the SUM, DSUM, and SWAP gates were examined in detail.

We have mainly considered the entangling power based upon the linear entropy. However, one is more interested in the entangling power based on the von Neumann entropy. Fortunately, the former provides a lower bound to the latter. In each of the cases we consider, our results show that for large dimension, to leading order the average entanglement created is equal to the maximum entanglement. Investigations of the entangling powers and operator entanglement will be helpful in understanding the entangling capabilities of quantum operations as physical resources, and will play an important role in quantum information theory.

\section{Acknowledgments}

We acknowledge valuable discussions with Paolo Zanardi, Jamil Daboul, and Stephen D Bartlett. This project has been supported by an Australian Research Council Large Grant and Macquarie University Research Fellowship. 
[2] P. Zanardi, C. Zalka, and L. Faoro, Phys. Rev. A 62, 030301(R) (2000).

[3] W. Dür, G. Vidal, J. I. Cirac, N. Linden, and S. Popescu, Phys. Rev. Lett. 87, 137901 (2001); B. Kraus, W. Dür, G. Vidal, J. I. Cirac, M. Lewenstein, N. Linden and S.Popescu, Z. Naturforsch. 56a, 91 (2001); J. I. Cirac, W. Dür, B. Kraus, and M. Lewenstein, Phys. Rev. Lett. 86, 544 (2001); K. Hammerer, G. Vidal, and J. I. Cirac, Phys. Rev. A 66, 062321 (2002); G. Vidal, K. Hammerer, and J. I. Cirac, Phys. Rev. Lett. 88, 237902 (2002); W. Dür, G. Vidal, and J. I. Cirac, Phys. Rev. Lett. 89, 057901 (2002).

[4] Y. Makhlin, quant-ph/0002045 (2000).

[5] M. S. Leifer, L. Henderson, and N. Linden, Phys. Rev. A 67, 012306 (2003).

[6] B. Kraus and J. I. Cirac, Phys. Rev. A 63, 062309 (2001); B. Kraus, K. Hammerer, G. Giedke, and J. I. Cirac, Phys. Rev. A, to appear; quant-ph/0210136 (2002).

[7] C. H. Bennett, A. W. Harrow, D. W. Leung, and J. A. Smolin, quant-ph/0205057 (2002).

[8] A. M. Childs, D. W. Leung, F. Verstraete, and G. Vidal, quant-ph/0207052 (2002).

[9] D. W. Berry and B. C. Sanders, quant-ph/0205181 (2002); quant-ph/0207065 (2002).

[10] M. M. Wolf, J. Eisert, and M. B. Plenio, quant-ph/0206171 (2002).

[11] M. A. Nielsen, C. M. Dawson, J. L. Dodd, A. Gilchrist, D. Mortimer, T. J. Osborne, M. J. Bremner, A. W. Harrow, and A. Hines, Phys. Rev. A, to appear; quant-ph/0208077 (2002).

[12] P. Zanardi, Phys. Rev. A 63, 040304(R) (2001).

[13] X. Wang and P. Zanardi, Phys. Rev. A, 66, 044303 (2002).

[14] J. L. Brylinski and R. Brylinski, quant-ph/0108062 (2001).

[15] M. J. Bremner, C. M. Dawson, J. L. Dodd, A. Gilchrist, A. W. Harrow, D. Mortimer, M. A. Nielsen, and T. J. Osborne, Phys. Rev. Lett. 89, 247902 (2002).

[16] D. Gottesman, quant-ph/9802007 (1998); quant-ph/9807006 (1998); D. Gottesman, A. Kitaev, and J. Preskill, Phys. Rev. A 64, 012310 (2001).

[17] G. Alber, A. Delgado, N. Gisin, and I. Jex, J. Phys. A 34, 8821 (2001).

[18] S. D. Bartlett, H. de Guise, and B. C. Sanders, Phys. Rev. A 65, 052316 (2002).

[19] B. C. Sanders, S. D. Bartlett, and H. de Guise, quant-ph/0208008 (2002).

[20] In Ref. 2], the entangling power was defined using a different notation $e_{\mathrm{p}}(U):=\overline{E\left(U\left|\psi_{1}\right\rangle \otimes\left|\psi_{2}\right\rangle\right)}$.

[21] T.-C. Wei, K. Nemoto, P. M. Goldbart, P. G. Kwiat, W. J. Munro, and F. Verstraete, Phys. Rev. A, to appear; quant-ph/0208138

[22] D. W. Berry and Barry C Sanders, unpublished.

[23] X. Wang, B. C. Sanders, and S. H. Pan, J. Phys. A 33, 7451 (2000).

[24] X. Zhou, D. W. Leung, and I. L. Chuang, Phys. Rev. A 62, 052316 (2000).

[25] D. Collins, N. Linden, and S. Popescu, Phys. Rev. A 64, 032302 (2001).

[26] J. Daboul, X. Wang, and B. C. Sanders, J. Phys. A, accepted; quant-ph/0211185 (2002).

[27] S. D. Bartlett, private communication.

[28] K. Fujii, quant-ph/0207002 v3 (2002).

[29] D. Gottesman and I. L. Chuang, Nature 402, 390 (1999).

[30] The SUM gate may create entanglement of $\ln d$ from the state $\sum_{n}|n\rangle|0\rangle$. This entanglement is the maximum because the SUM gate may be implemented nonlocally using this much entanglement via a generalization of the Gottesmann-Chuang [29] gate. Both the DSUM and SWAP gates create entanglement of $2 \ln d$ from initial states where the qudits acted upon are maximally entangled with ancillas; this entanglement is the maximum possible for a system of this dimension. Similarly, without ancillas the maximum possible entanglement is $\ln d$, and this may be created via the DSUM gate from the initial state $|0\rangle \sum_{n}|n\rangle$. 